\begin{tabular}{|c|l|}
\hline Title & Elasticity of Living Cells on a Microarray during the Early Stages of A dhesion Measured by A tomic Force Microscopy \\
\hline Author(s) & $\begin{array}{l}\text { Mizutani, Y usuke; T suchiya, Masahiro; Hiratsuka, Shinichiro; Kawahara, Koichi; Tokumoto, Hiroshi; Okajima, } \\
\text { Takaharu }\end{array}$ \\
\hline Citation & $\begin{array}{l}\text { Japanese Journal of A pplied Physics, 47(7),6177-6180 } \\
\text { https://doi.org/40.1143/JJAP.47.6177 }\end{array}$ \\
\hline Issue Date & 2008-07 \\
\hline Doc URL & http://hdl.handle.net/2115/45746 \\
\hline Type & article(author version) \\
\hline File Information & JJA P47_6177-6180.pdf \\
\hline
\end{tabular}

Instructions for use 


\section{Elasticity of Living Cells on a Microarray during the Early Stages of Adhesion Measured by Atomic Force Microscopy}

\section{Authors:}

Yusuke MIZUTANI, Masahiro TSUCHIYA, Shinichiro HIRATSUKA, Koichi KAWAHARA, Hiroshi TOKUMOTO ${ }^{1}$, and Takaharu OKAJIMA*

\section{Address:}

Graduate School of Information Science and Technology, Hokkaido University, Japan.

${ }^{1}$ Nanotechnology Research Center, Research Institute for Electronic Science, Hokkaido University, Japan

* Corresponding author

Address: Kitaku N14 W9, Sapporo, 060-0814, Japan

Email: okajima@ist.hokudai.ac.jp

Tel/Fax: +81-(0)11-706-7698 


\begin{abstract}
The number distribution of the elastic modulus of fibroblast cells was successfully measured during the early stages of adhesion using an atomic force microscope (AFM) combined with a microarray as a substrate, which allowed us to arrange and culture the cells so that a large number of cells could be measured for a short time. We confirmed that cells deposited in the wells of the microarray could be cultured for at least $12 \mathrm{~h}$ without any significant migration. Histograms of the Young's modulus, E, of cells during the early stages of adhesion produced from force curve measurements of cells ( $n=\sim 300$ ) cultured for 3-9 h were well fitted to a log-normal distribution. With increasing incubation time, the average value of $E$ increased significantly, while the standard deviation of the distribution remained almost constant. The results are discussed in terms of the cytoskeleton inside cells.
\end{abstract}

KEYWORDS: atomic force microscope, microarray, fibroblast cells, Young’s modulus, log-normal distribution 


\section{Introduction}

Some types of cells adhere to their surrounding extracellular matrices and/or neighboring cells in vivo and subsequently display various functions such as motility and division. When suspended adhesive cells are placed on a solid substrate in vitro, they adhere to the substrate and alter their morphology and gene expression, which are considered to be strongly related to the cellular mechanics governed by the internal stiffness of the cytoskeleton assembly ${ }^{1}$. The micro- and nanomechanics of cells adhered tightly to substrates have been widely investigated using particle tracking techniques, ${ }^{2-6)}$ microfabricated substrates, ${ }^{1,7)}$ and atomic force microscopy (AFM). ${ }^{8)}$

AFM has the advantages of allowing us to measure the mechanical properties of cells at any region of the surface by controlling the position of the AFM probe and not requiring any modification of the cell surface or substrate. Previous AFM studies ${ }^{9-13)}$ have revealed heterogeneity in the elasticity of single cells. Moreover, the viscoelastic properties of cells have been measured in the force modulation mode, ${ }^{13-16)}$ which is the basis of measurements in a frequency domain, and in the stress relaxation mode ${ }^{17,18)}$ in a time domain.

On the other hand, little is known about how suspended cells attain the metastable state in which they adhere tightly to substrates. At an early stage of adhesion, filamentous structures, such as F-actin, microtuble and intermediate filaments, may be formed and modified more drastically inside cells, and therefore measurements of the mechanical properties of cells during the early stages of adhesion provide information 
about the kinetics of the formation of internal structures that affect the cellular mechanics.

Since cells are fragile during the early stages of adhesion and only adhere weakly to substrates, techniques for preventing the detachment of cells from substrates are required. Moreover, it is considered that cells exhibit temporal fluctuations as well as individual differences during adhesion, and therefore large numbers of cells need to be measured rapidly in order to estimate their cellular mechanics in a quantitative manner.

Here, we present a new method of AFM force measurements combined with a microarray technique. ${ }^{19)}$ In the method, cells are placed and cultured in the wells of a microarray, and force measurements of the cells are automatically examined at the centers of the wells without confirming the positions of all the cells. Using this method, we measured the number distribution of the elastic modulus of mouse fibroblast cells as a function of the incubation time and discuss the obtained results in terms of the internal structures of the cytoskeleton.

\section{Experimental Procedure}

\subsection{Microarray}

We used a commercial cell microarray (Livecell array ${ }^{\mathrm{TM}}$; Nunc Co., USA), which comprises a hexagonal structure of microwells with a depth of $\sim 8 \mu \mathrm{m}$ and spacing of 20 $\mu \mathrm{m}$ fabricated on a glass substrate. The substrate was precoated with fibronectin (BD Bioscience, USA). 


\subsection{Cell samples}

Mouse fibroblast NIH3T3 cells were cultured in Dulbecco’s modified Eagle’s medium (DMEM; Sigma, USA) containing penicillin (100 units/mL)-streptomycin (100 mg/mL) (Sigma, USA) and 10\% fetal bovine serum (GIBCO-BRL Life Technologies, USA) in culture dishes at $37^{\circ} \mathrm{C}$ in a $5 \% \mathrm{CO}_{2}$ atmosphere for $1-2 \mathrm{~d}$. After suspension with trypsin (Sigma, USA), the cells were deposited on the microarray in complete medium (DMEM containing FBS) and immediately incubated for $t_{\mathrm{c}}=3,6$ or $9 \mathrm{~h}$ under the same conditions described above. The total number of suspended cells exceeded the number of wells, such that all the wells of the microarray were occupied by cells. The cells were cultured for the appropriate times, washed with PBS, placed in $5 \mathrm{~mL}$ of $\mathrm{CO}_{2}$-independent medium (GIBCO-BRL Life Technologies, USA) and immediately used for AFM experiments.

\subsection{Immunofluorescence observation}

Immunofluorescence imaging of the nuclei and actin molecules in cells cultured in the microarray was performed with a confocal laser scanning microscope (CLSM) (FV-300; Olympus, Japan). After removing the medium from the microarray, the cells were fixed with $4 \%$ formalin in phosphate-buffered saline (PBS, $\mathrm{pH}$ 7.2) for $30 \mathrm{~min}$, rinsed three times with PBS, permeabilized in $0.01 \%$ Triton X-100 for 10 min and rinsed three times with PBS. Next, rhodamine-phalloidin (Molecular Probes, USA) in PBS was added and the cells were incubated for $1 \mathrm{~h}$ at $37^{\circ} \mathrm{C}$. Excess dye was removed by rinsing the cells three times with PBS. To prevent rapid photobleaching and stain 
the nuclei, VECTASHIELD ${ }^{\circledR}$ mounting medium containing DAPI (Vector Laboratories, USA) was used.

\subsection{AFM measurements}

We used a commercial AFM (MFP-3D AFM; Asylum Research, USA) mounted in an inverted optical microscope (TE-2000E; Nikon, Japan) (see Fig. 1). A small rectangular cantilever (BL-AC40TS; Olympus Co., Japan) with a nominal spring constant of $0.1 \mathrm{~N} / \mathrm{m}$ was used. A colloidal silica bead with a radius of $5 \mu \mathrm{m}$ (Funakoshi, Japan) was attached to the apex of the AFM tip ${ }^{20)}$ using an epoxy glue to measure the elastic modulus of the cells in a more quantitative manner. Prior to cell experiments, the spring constant of the cantilever was determined using a thermal fluctuation method. ${ }^{21)}$ The loading force was determined by multiplying the calibrated cantilever spring constant by its deflection on the basis of Hooke's law.

Topographic images and force measurements were obtained using a force mapping technique. ${ }^{8)}$ In the topographic imaging, force curves were taken at intervals of $2.5 \mu \mathrm{m}$, and the contact positions estimated from the force curves were imaged. In the force measurements, force curves were taken within $1 \mathrm{~h}$ at the centers of the wells, which numbered more than 300 in a cell sample using a software control. The maximum loading force and the scan speed were approximately $450 \mathrm{pN}$ and $15 \mu \mathrm{m} / \mathrm{s}$, respectively. 
The apparent Young's modulus, E, of cells was estimated by fitting the force curves obtained during the approach process at the centers of the wells to a Hertz model, which was expressed by

$$
F=\frac{4}{3} \frac{E}{1-v^{2}} R^{1 / 2} \delta^{3 / 2}
$$

where $F$ is the loading force, $v$ is the Poisson's ratio, $\delta$ is the indentation depth of the probe, and $R$ is the radius of the probe to be $5 \mu \mathrm{m}$. The $v$ was assumed to be 0.5 .

\section{Results}

Figure 2(a) shows a fluorescence image of the nuclei of NIH3T3 cells cultured for $t_{\mathrm{c}}=12 \mathrm{~h}$ on the microarray. The positions of the cell nuclei were in good agreement with those of the wells. Moreover, we confirmed that there was no overlapping of nuclei by changing the focal plane of the CLSM in the $\mathrm{z}$ direction. Since NIH3T3 cells are mononuclear, these observations indicated that the cells were arranged as a monolayer in the wells of the microarray. The filling rate of cells to wells was estimated to be $>98 \%$ from a fluorescence image of the nuclei on a large scale.

Figures 2(b) and (c) show fluorescence images of F-actin staining in cells cultured for $t_{\mathrm{c}}=3$ and $12 \mathrm{~h}$ on the microarray, respectively. Although the filamentous structures of F-actin were clearly visualized for both incubation times, thick filaments that probably represented stress fibers were observed for $t_{\mathrm{c}}=12 \mathrm{~h}$, but not for $t_{\mathrm{c}}=3 \mathrm{~h}$. The results shown in Fig. 2 revealed that the microarray was useful for retaining and culturing cells in the wells without losing their adhesive function. 
Figure 3 shows topographic images of cells cultured on the microarray for different periods of time. Since a colloidal probe was used for imaging, the obtained topographic images had low spatial resolution. However, the images were stably obtained, indicating that the cells were stable in the wells with no detachment from the substrate during the force curve measurements. As shown in Fig. 3(a), the cells were spherical at $t_{\mathrm{c}}=3 \mathrm{~h}$. Since the suspended cells were spherical with similar sizes in Fig. 3(a), we concluded that the cell morphology remained almost unchanged within $t_{\mathrm{c}}=3 \mathrm{~h}$.

As shown in Fig. 3(b), the cells remained spherical at $t_{\mathrm{c}}=6 \mathrm{~h}$. However, when the cells were incubated for $9 \mathrm{~h}$, they exhibited drastic changes in shape and became asymmetrical and flatter, while the spacing of the cells remained the same as that of the wells. The finding that the cells began to expand in the wells without any significant migration allowed us to estimate the elastic modulus of the cells automatically by conducting force curve measurements at the centers of the wells.

Figures 4(a) to (c) show histograms of the Young's modulus, E, of the cells in the wells of the microarray during the early stages of culture. Histograms of the logarithms of $E$ were found to be well fitted to a Gaussian function, that is, the histograms exhibited a log-normal distribution. Figure 5 shows the average values and standard deviations of $E$ estimated from Figures 4(a) to (c) as a function of the incubation time. The average value of $E$ increased significantly with increasing incubation time, while the standard deviation of the distribution remained constant.

\section{Discussion}


Previous studies ${ }^{4-6)}$ of cells adhered tightly to substrates have revealed that the rocking amplitudes at frequencies corresponding to the elastic components obtained using magnetic twisting cytometry and laser tracking microrheology exhibited log-normal distributions. Balland et al. ${ }^{4)}$ proposed a model to explain why the rocking amplitudes displayed log-normal distributions. Briefly, they modeled the cytoskeleton network as an infinite assembly of an elementary unit with viscoelastic properties. In the present study, we observed that the cells became flatter during the formation of a thick filamentous network, suggesting that the formation of a cytoskeleton network induced the increases in $E$ with a log-normal distribution.

Interestingly, even for $t_{\mathrm{c}}=3 \mathrm{~h}$ when the cytoskeleton network seemed to be insufficiently developed, the histogram of $E$ exhibited a clear log-normal distribution. Guck et al. ${ }^{22)}$ measured suspended fibroblast cells after trypsinization to detach the cells from a dish. They found that the density of the cytoskeleton inside the cells exhibited a log-normal distribution using fluorescence confocal microscopy experiments. We found that the amount of F-actin was abundant inside the cells after $3 \mathrm{~h}$ of incubation, implying that the density of F-actin, which is one of the major contributors to the elastic modulus of cells measured by $\mathrm{AFM}^{11)}$, reflects the histogram of $E$ shown in Fig. 4(a).

If one can accept that two factors, namely the formation of the cytoskeleton network and the density of F-actin, may lead to the log-normal distribution of the observed Young's modulus of the cells, the fitting to a single Gaussian function presented in Fig. 4 may be a rough approximation. Detailed investigations of the 
cellular mechanics, including the viscoelastic properties, should be required to elucidate the origin of the log-normal distribution during the early stages of adhesion.

The average value of $E$ for $t_{\mathrm{c}}=9 \mathrm{~h}$ was ca. $250 \mathrm{~Pa}$, which was smaller than that estimated for NIH3T3 cells on a flat substrate in a previous study. ${ }^{11)}$ The difference may arise from the incubation time and the cell density. In the former, it was considered that the cells for $t_{\mathrm{c}}=9 \mathrm{~h}$ were in a transient state while attaining their metastable state for tight adherence to the substrates. In the latter, it was considered that the cell density on the microarray was much higher than that on the flat substrate in the previous study ${ }^{11)}$, and that this difference in the cell density altered the kinetics of the formation of the cytoskeleton network and of the polymerization/depolymerization of actin, tubulin and intermediate filaments due to cell-cell interactions, although both the isolated cells adhered on the flat substrate and the cells on the microarray were not in their native environments.

\section{Conclusions}

The number distribution of the Young's modulus, E, of fibroblast cells ( $\mathrm{n}=\sim 300$ ) was successfully measured during the early stage of adhesion by AFM. Cells were placed in the wells of the microarray to measure the elastic modulus as a function of the incubation time. The results revealed that the histograms of $E$ of the cells during the early stages of adhesion were well fitted to a log-normal distribution. The average value of $E$ increased significantly as the incubation time increased, without any significant change in the standard deviation. We believe that this technique of AFM 
combined with a microarray will be useful for investigating the statistics of cellular mechanics with temporal fluctuations.

\section{Acknowledgements}

This work was supported by an Industrial Technology Research Grant Program in 2006 from the New Energy and Industrial Technology Development Organization (NEDO) of Japan. 


\section{References}

1) D. E. Ingber: Int. J. Dev. Biol. 50 (2006) 255.

2) Y. Tseng, T. P. Kole, and D. Wirtz: Biophys. J. 83 (2002) 3162.

3) B. Fabry, G. N. Maksym, J. P. Butler, M. Glogauer, D. Navajas and J. J. Fredberg: Phys. Rev. Lett. 87 (2001) 148102

4) M. Balland, N. Desprat, D. Icard, S. Féréol, A. Asnacios, J. Browaeys, S. Hénon and F. Gallet: Phys. Rev. E 74 (2006) 021911

5) B. D. Hoffman, G. Massiera, K. M. Van Citters and J. C. Crocker: Proc. Natl. Acad. Sci. U.S.A. 103 (2006) 10259.

6) G. Massiera, K. M. Van Citters, P. L. Biancaniello, and J. C. Crocker: Biophys. J. 93 (2007) 3703.

7) J. L. Tan, J. Tien, D. M. Pirone, D. S. Gray, K. Bhadriraju, and C. S. Chen: Proc.Natl.Acad.Sci.U.S.A 100 (2003) 1484.

8) B. P. Jena and J. K. H. Horber Ed. 2002 Atomic force microscopy in cell biology, Methods in Cell Biology 68 (Academic Press)

9) J. H. Hoh and C. A. Schoenenberger: J. Cell Sci. 107 (1994) 1105.

10) C. Rotsch, K. Jacobson and M. Radmacher: Proc.Natl.Acad.Sci.U.S.A 96 (1999) 921.

11) C. Rotsch and M. Radmacher: Biophys. J. 78 (2000) 520.

12) E. A-Hassan, W. F. Heinz, M. D. Antonik, N. P. D`Costa, S. Nageswaran, C. A. Schoenenberger and J. H. Hoh: Biophys. J. 74 (1998) 1564.

13) M. Radmacher, R. W. Tillmann, M. Fritz and H. E. Gaub: Science 257 (1992) 1900. 
14) R. E. Mahaffy, C. K. Shih, F. C. MacKintosh, and J. Kas: Phys. Rev. Lett. 85 (2000) 880.

15) J. Alcaraz, L. Buscemi, M. Grabulosa, X. Trepat, B. Fabry, R. Farre and D. Navajas: Biophys. J. 84 (2003) 2071.

16) B. A. Smith, B. Tolloczko, J. G. Martin and P. Grutter : Biophys. J. 88 (2005) 2994.

17) E. M. Darling, S. Zauscher, J. A. Block and F. Guilak: Biophys. J. 92 (2007) 1784.

18) T. Okajima, M. Tanaka, S. Tsukiyama, T. Kadowaki, S. Yamamoto, M. Shimomura and H. Tokumoto: Nanotechnology 18 (2007) 084010.

19) M. Deutsch, A. Deutsch, O. Shirihai, I. Hurevich, E. Afrimzon, Y. Shafran and N. Zurgil: Lab Chip 6 (2006) 995.

20) T. Okajima, M. Tanaka, S. Tsukiyama, T. Kadowaki, S. Yamamoto, M. Shimomura and H. Tokumoto: Jap. J. Appl. Phys. 46 (2007) 5552.

21) J. L. Hutter and J. Bechhoefer: Rev. Sci. Instrum. 64 (1993) 1868.

22) J. Guck, S. Schinkinger, B. Lincoln, F. Wottawah, S. Ebert, M. Romeyke, D. Lenz, H. M. Erickson, R. Ananthakrishnan, D. Mitchell, J. Kas, S. Ulvick and C. Bilby: Biophys. J. 88 (2005) 3689. 


\section{Figure captions}

Fig. 1. Schematic diagram of an AFM combined with a microarray, on which living cells were deposited. The optical microscope was employed to choose a particular cell and to set the cantilever tip at the center of well before successive force curve measurements.

Fig. 2. (a) Micrograph of NIH3T3 cells deposited in the wells of the microarray and cultured for $12 \mathrm{~h}$. The cells were tagged by staining their nuclei with DAPI. Immunolabeling of F-actin in cells in the wells of the microarray after incubation for (b) $3 \mathrm{~h}$ and (c) $12 \mathrm{~h}$. The scale bars are $20 \mu \mathrm{m}$.

Fig. 3. Topography of NIH3T3 cells measured in the force mapping mode. The incubation times were $3 \mathrm{~h} \mathrm{(a),} 6 \mathrm{~h}$ (b) and 9 h (c). The horizontal scale bars are $20 \mu \mathrm{m}$. The height scale is relative among the images since the absolute position of the substrate could not be measured.

Fig. 4. Histogram of the Young's modulus, E, of cells in the wells of the microarray after incubation for $3 \mathrm{~h} \mathrm{(a),} 6 \mathrm{~h}$ (b) and $9 \mathrm{~h}$ (c). The histograms were fitted to a single log-normal distribution function.

Fig. 5 Average values and standard deviations of $E$ estimated from the log-normal distribution shown in Fig. 4 as a function of the incubation time. 
Fig. 1 Mizutani et al.

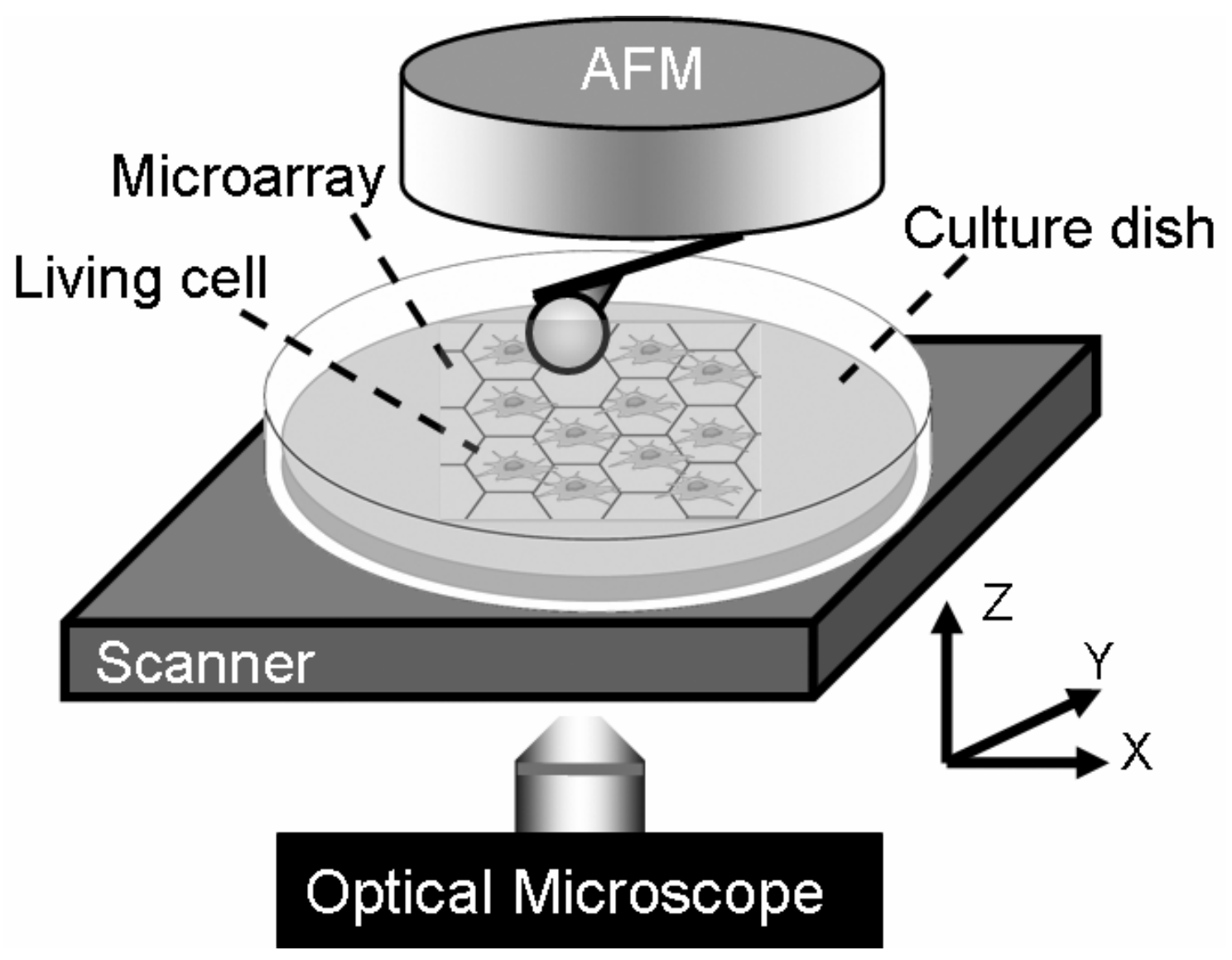


Fig. 2 Mizutani et al.
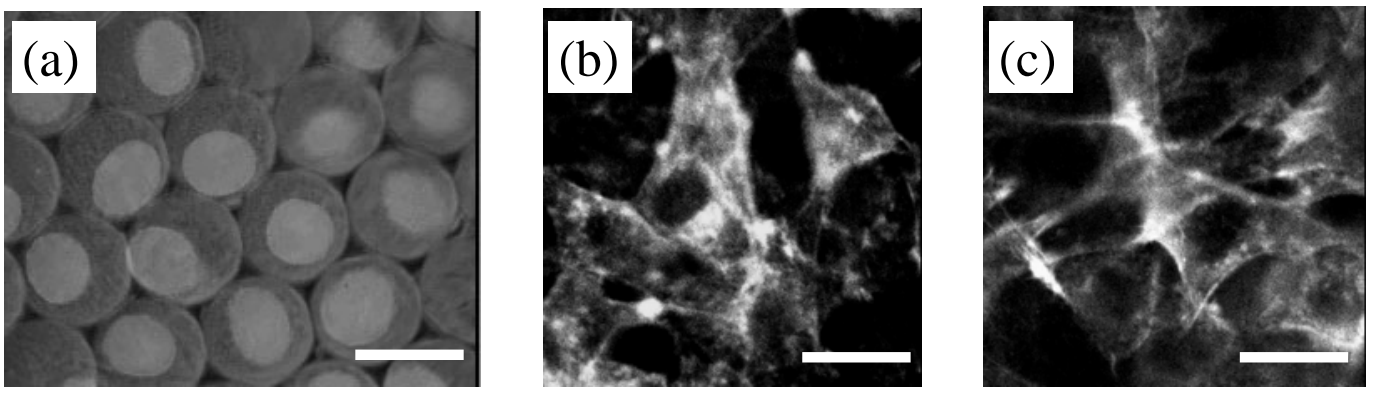
Fig. 3 Mizutani et al.
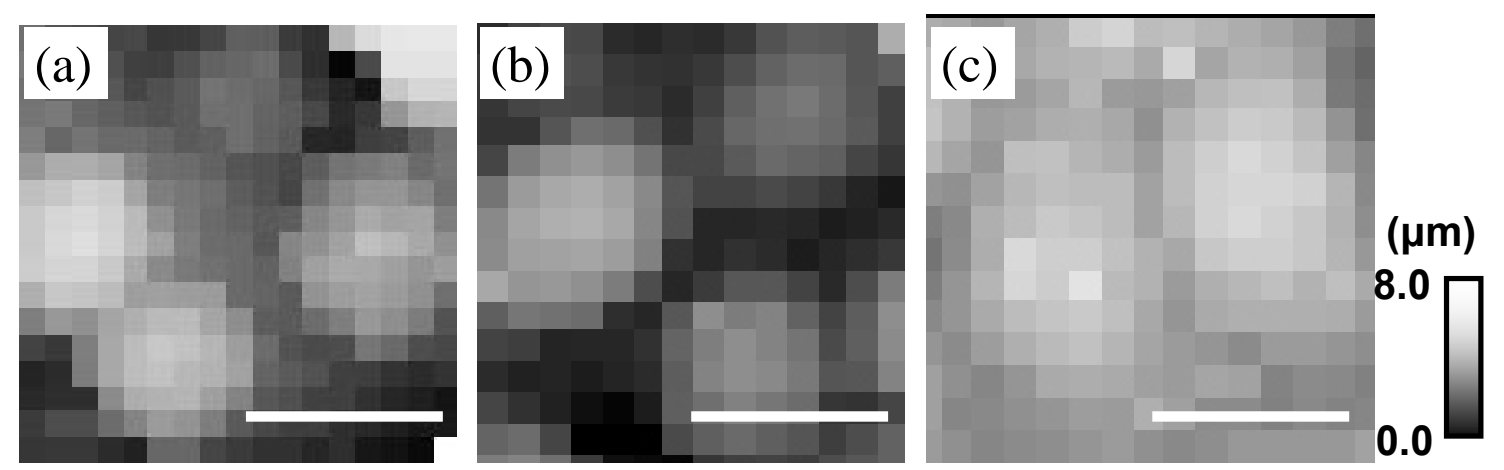
Fig. 4 Mizutani et al.

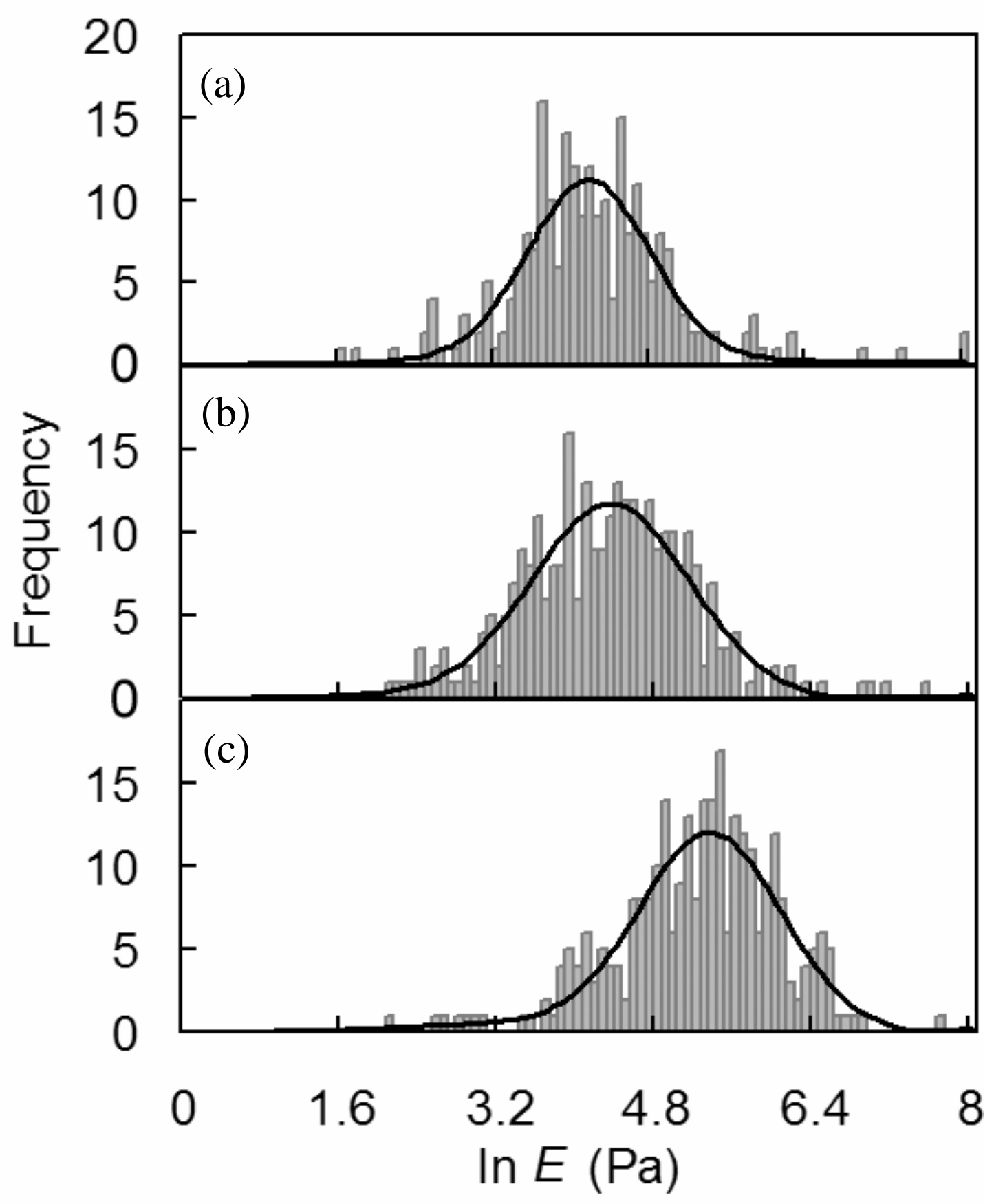


Fig. 5 Mizutani et al.

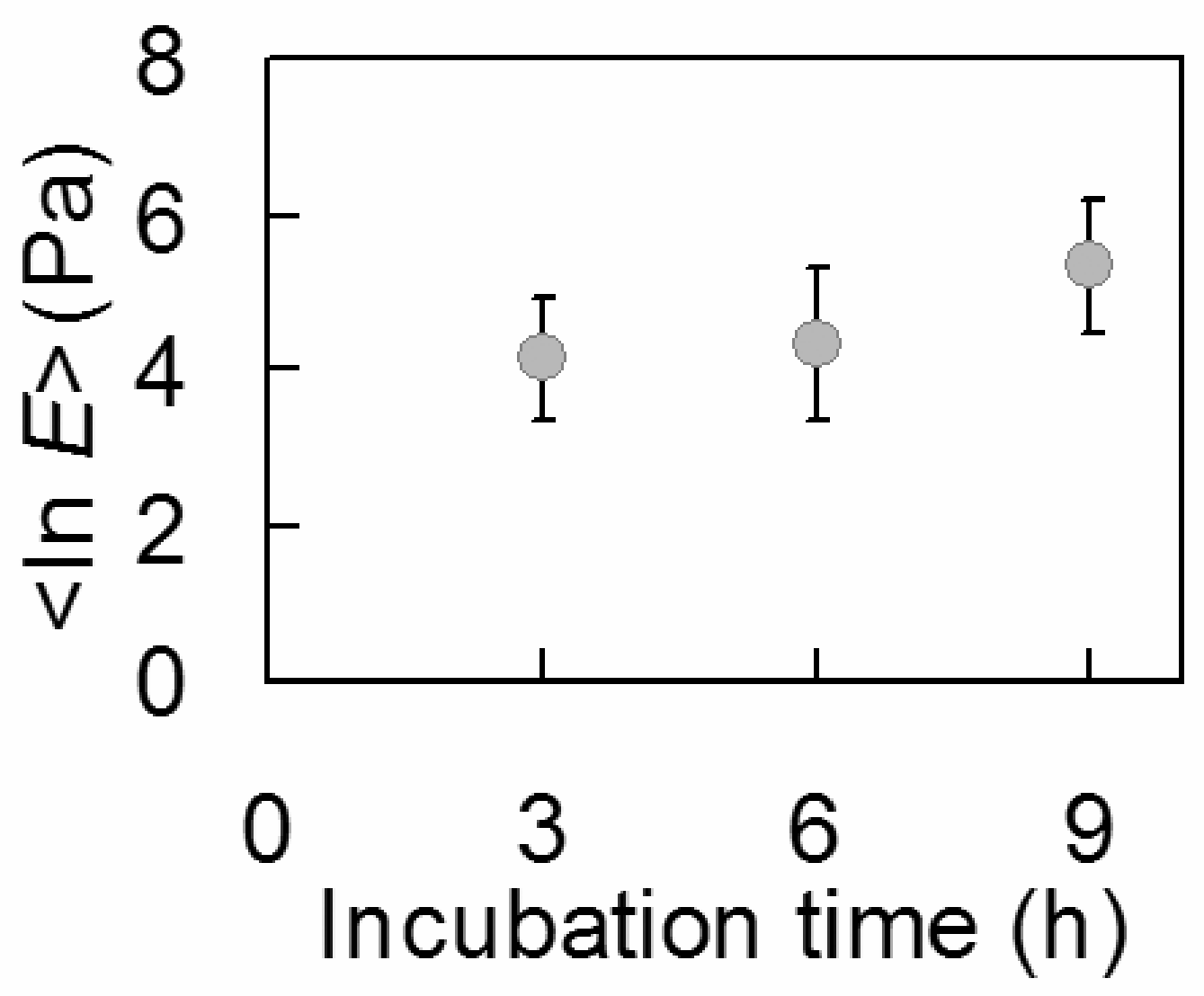

